\title{
From a forecasting methodology for the electric energy consumption of mono-towns to its sustainability
}

\author{
S. V. Fedorova ${ }^{1}$, P. Yu. Khudyakov ${ }^{2}$, N. A. Melkozerov ${ }^{2}$ \\ \& D. A. Firsova ${ }^{2}$ \\ ${ }^{1}$ Technical University of UGMK, Russia \\ ${ }^{2}$ Ural Federal University, Russia
}

\begin{abstract}
The need of Russia for a resource-innovative energy strategy for the period up to 2035 makes it urgent to create a methodology of strategic forecasting for the electric energy consumption of mono-towns, which will result in the efficient development of a power supply system, ensuring territories' sustainability.

For this purpose, it is necessary to consider a mono-town as a complete system, a living organism with a definite life cycle and structure of energy consumers. The authors offer a technocoenosis approach to carry out the ranking and structural analysis of the electric energy consumption of one of the Sverdlovsk region's mono-towns, taking into account dynamics in population, enterprises, organizations and institutions for the period of 5 years.

The provided analysis makes it possible to judge the technocoenosis optimality using characteristic exponent $\beta$, depending on the structural features of the territory.

The authors developed an algorithm for electric energy consumption forecasting, based on Support Vector Machines (SVM), which takes into account the electric energy of mono-towns' consumers and the climatic factors.

Forecasting accuracy was achieved using cross-validation of the input data in order to optimize the training model.

The corresponding changes in electric energy consumption, when a characteristic exponent is optimal, will result in a target forecast that provides the sustainable development of mono-towns.
\end{abstract}

Keywords: strategy, forecasting algorithm, electric energy consumption, technocoenosis approach, mono-town sustainability. 


\section{Introduction}

According to the Russian energy strategy for the period up to 2035, the "Energy policy will be redirected from resource-oriented to resource-innovative development, highlighting the quality changes in power consumption structure, energy service improvement, technological energy-saving and modernization, advanced electrification , rather than quantitative increase of volume terms...".

Any given line of energy development requires new methodology development. The methodology deals with power-efficiency management across the functionally territorial establishments, mono-towns in particular.

The data of the Expert Institute (Moscow) is presented in the monograph originated by Uskova et al. [1]. According to that data, 467 towns and 332 villages were listed as mono-profile settlements with a population of 24,544 thousand people, that is $17.2 \%$ of the Russian commonwealth. More than 900 city-forming enterprises function in the territory; these enterprises produce more than $30 \%$ of Russian industrial products volume [1].

In compliance with developed criteria, a town should be considered as mono-town on the condition that the population of the town counts more than 3 thousand people and more than $20 \%$ of able-bodied citizens work at a city-forming enterprise.

In the Sverdlovsk region, more than $40 \%$ of functionally territorial establishments can be defined as mono-towns.

That is why the problem of modification methods for mono-towns, providing the self-development ability of mono-towns and adaptability concerning external medium transformations demands development of a new qualitative approach. These approaches deal with new controlling mechanisms development, the mechanisms monitoring sustainable development processes in mono-specializing towns; this is the most important condition, enabling sustainable further development of the region's economy and the Russian economy as a whole.

Efficient energy consumption and efficient energy utilization determine the achievement of principal indices, the indices connected with power efficiency improvement.

On the basis of the analysis of scientific sustainable development approaches, including those of mono-towns [2-7], the authors developed a strategic forecasting algorithm using objective technocoenosis sustainability laws [8-11]. A strategic forecasting algorithm is a sustainability development instrument for energy supply systems and energy consumption systems in mono-towns.

\section{Sustainable development issues at mono-towns}

In accordance with UNO. documents, a town can be considered as sustainable if "social, economic and physical development are permanent; town has to be supplied with natural resources; long-term community safety must be provided, including safety at a time of ecological and natural disasters" [4]. 
As Kuvshinov [6] put it, sustainable territory development is the purposeful monitoring process in a municipal union socio-economic system; the process provides stability of system links, system elements and system structure as a whole, in line with standard of life improvement, in the context of environmental and ecological balance. Attention is attracted to the superior conception determination, the conception leads to sustainable system formation. In compliance with the information mentioned above, a system has to be able to steadily develop, be self-controlling, self-ruling, self-improving, and use mostly internal resources, as well as the Earth's resources [6].

According to the definition given by Ikonnikova [7], the evolutional nature of sustainable territory development leads to both citizen prosperity and potential improvement of the territory for future generations.

Martynov et al. [12] hold that sustainable territory development is a fundamental system attribute. Three subsystems had been marked out, namely: natural resources, material and technical complex and social medium. In addition, it is pointed out that development sustainability as a system attribute can be applied to each of the subsystems. At the same time, material system stability depends on three parameters, to be exact: extensive (power-substantial potential), intensive (reproduction and interchange processes) and informational (composition and structure) [12].

System sustainability is developed as an ability to maintain stability under various indignations and to optimize parameters, maintaining the structure, as stated by Fufaev [9] and Kudrin [10]. It is proved that "sustainability is formed by self-organization, internal and external sphere factors totality in multi-aspectual community of organizations (towns, districts, regions, republics and country all in all): the less the organization (working in one activity category) number, the more such activities and vice versa, as the number of organizations working in one activity category increase, the quantity of these activities decrease" [9].

Having analyzed the nature of dynamic electricity consumption, a dominating dependence had been detected in the example of a mono-town located in the Sverdlovsk region (fig. 1).

The development instability of mono-towns is connected with production volume shortening or the closing of city-forming enterprises on the one hand and the legal potential of an alternative economy (small-scale business) on the other hand; this can be detected in the rank and structural analysis of energy consumption.

Investigations were carried out on the "Basis element" demand in 18 monotowns and small towns by the Strategic Development Center in 2013. Three typical models were organized dealing with the supply and development of mono-towns.

The model referred to as "Guided contraction" can be applied in relation to mono-towns with closable city-forming enterprises and with no opportunity to improve the city's economic growth; the "Stable mono-town model" - used for towns with no potential for alternative economic development, but with stable working city-forming enterprises; the "Industrial diversification model" - this model is intended for cities that have potential for alternative branches of development. 

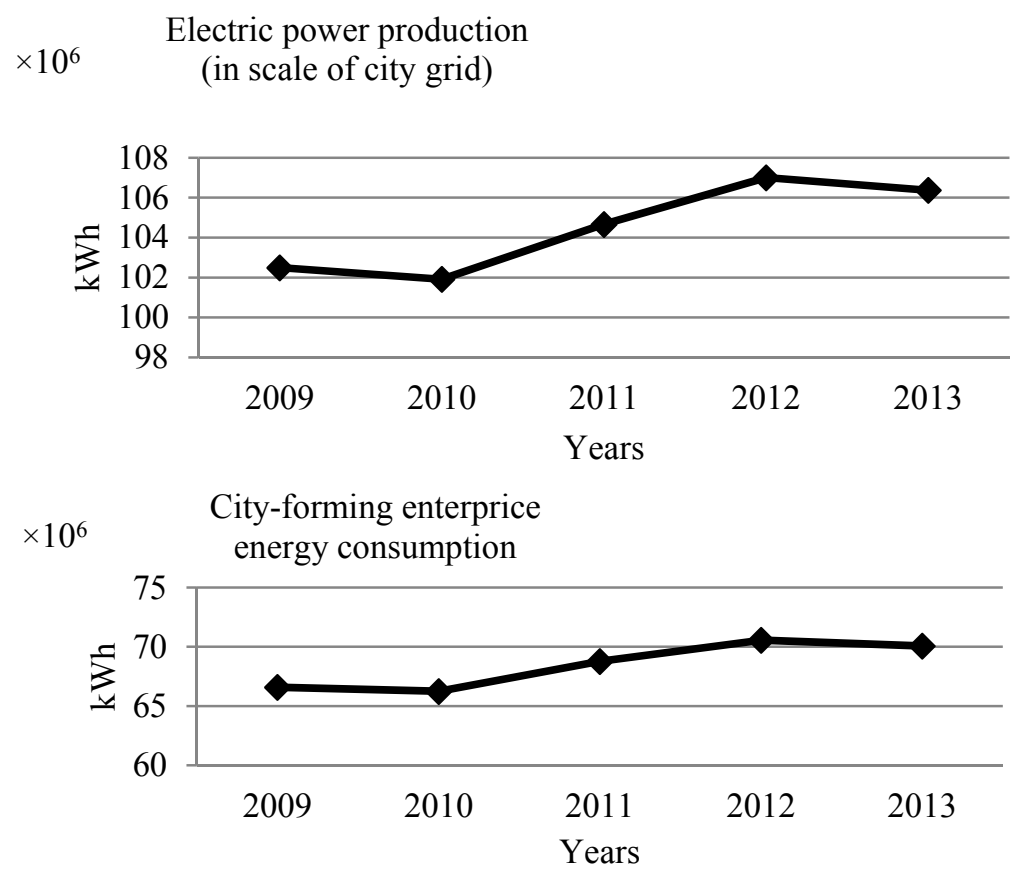

Figure 1: Energy consumption dynamics of a mono-town located in the Sverdlovsk region.

The model development that will be organized using an industrial park was proposed for Krasnoturyinsk.

The offered models should be considered as salvation programs against crisis situations at mono-towns.

Strategic development monitoring of a territory should be determined with the proper stage of territory evolution: ontogeny or succession [8].

Therefore, a mono-town is considered as technocoenosis, with a dedicated and formalized objective reality; a mono-town is characterized with links that have different bond strengths (strong, medium and predominantly weak). This is predicated upon the concept of sustainable economic development, on the basis of the evolutional economics changing theory of Nelson and Winter [24], Veblen [25], Popkov et al. [26], the achievements of the evolution theory belonging to Meyen [29], Mayewskiy [27], Chaitun [30], Chaikovskiy [28] and the technocoenosis theory defined by Kudrin [10], Fufaev [9] and Gnatyuk [8].

In order to overcome a crisis, a revolutionary method can be chosen through succession (innovative enterprise opening, application of distributed generation, smart greed, exploitation of new energy sources and introduction of intellectual facilities); this results in techocoenosis changing and creating the required conditions for sustainable development. 
At present, it is necessary to create a stable development model for remote mono-towns with underdeveloped infrastructures. The most appropriate way to do this is to correct the technocoenosis structure (energy consumers' structure and power equipment structure). For this purpose, a rank and structural analyzing method of energy consumption based on the technocoenosis approach is used.

\section{Rank and structural analysis of energy consumption in relation to mono-towns}

Deterministic systems (electric drive, electric power station) have hard cause-effect links between elements. In contrast to deterministic systems and Gaussian probabilistic and static objects (gas molecules), technocoenosis is selfregulating; a non-Gaussian system with slight internal connections, and technocoenosis is formed in conditions of limited resources. Therefore, internal or external resource (quantitative and structural) changes will probably result in mono-town instability.

The structural and rank analysis of energy consumption enables us to uncover changes taking place in technocoenosis.

The above-mentioned method is based on static data representation as rank allocation using energy consumption parameters for no less than 5 years.

The energy consumption of a single object is not regarded as isolated when the technocoenosis approach is used. It is considered as correlating with the rest, which are hierarchically systemized.

The methodology of rank allocation structure analysis lies in:

1. The main elements (enterprises, organizations, establishments, population the energy consumers) and the parameter under investigation (annual energy consumption) are singled out in technocoenosis (mono-town).

2. The consumers are ranged in the amount of energy consumed in descending order. The first rank is given to the object having the maximum parameter.

Rank H-allocation is described by the mathematical expression:

$$
W(r)=W_{1} r^{\beta},
$$

where $\mathrm{r}$ - object rank, $\mathrm{W}_{1}$ - the amount of energy consumed by the biggest object, $\beta$ - characteristic exponent determining the degree of slope of the $\mathrm{H}$-allocation curve [4].

The value of the characteristic exponent $\beta$ (liminal diapason of technocoenosis sustainability: $0.5 \leq \beta \leq 1.5$ ) reflects the degree of sustainability of territorial power equipment structure and technocoenosis evolutional development stage [2-4].

The authors perform the structural and rank analysis of energy-consumption in the context of both the Sverdlovsk region municipalities and mono-towns for the period of 2007-2013.

By the example of $\mathrm{H}$-allocations during the 2011 year, considering the energy consumption parameter, the characteristic exponent beta $\beta=2.4$. This means that 
a low growth rate in small towns occurs and there is a great gap between the megalopolis and other region municipalities [15].

$\beta<<0.5$ for mono-towns where there are closing city-forming enterprises, town infrastructure is poor and alternative business is not developed. This is a clear signal that technocoenosis is not formed from the point of view of evolutional diversity of species.

$\beta=1.4$ for mono-town, where there are 4 city-forming enterprises.

The results of the analysis of the power equipment structure which was carried out by the authors can be used as an example of the transformers located in monotowns' substations and are given below.

In compliance with the technocoenosis theory, for the investigation, transformers have been chosen with definite capacities S, kVA.

Hyperbolical rank $\mathrm{H}$-allocation with the approximating function construction (fig. 2) permits to define the characteristic exponent $\beta=1.8$. It means that quantitative deviations take place in technocoenosis itself, with the benefit of a "big" and "small" ratio position, insufficient quantity of low power transformers and excess of transformers with capacity $250 \mathrm{\kappa VA}, 160 \kappa \mathrm{VA}$.

In order to correct the quantitative technocoenosis structure, the analysis of the transformers' economic life and capacity factors was made for revealing the transformer types (fig. 3). On the basis of the analysis, replacement suggestions were calculated and advice was developed regarding the sustainable state maintaining of technocoenosis.

Regarding transformers with a nominal capacity of $400 \mathrm{kVA}$, lifetime analysis and capacity factor analysis was carried out. As a result, the following conclusion was made: it will be appropriate to replace the part of such transformers (more than $20 \%$ of transformers have life-time counts for 46 years, winter capacity factor $=0.34$; summer capacity factor $=0.17$ ). Transformers that have a smaller capacity should be installed instead of using outmoded equipment. Such replacements will create the ability to increase the sustainability of a towns' incoming electrical supply.

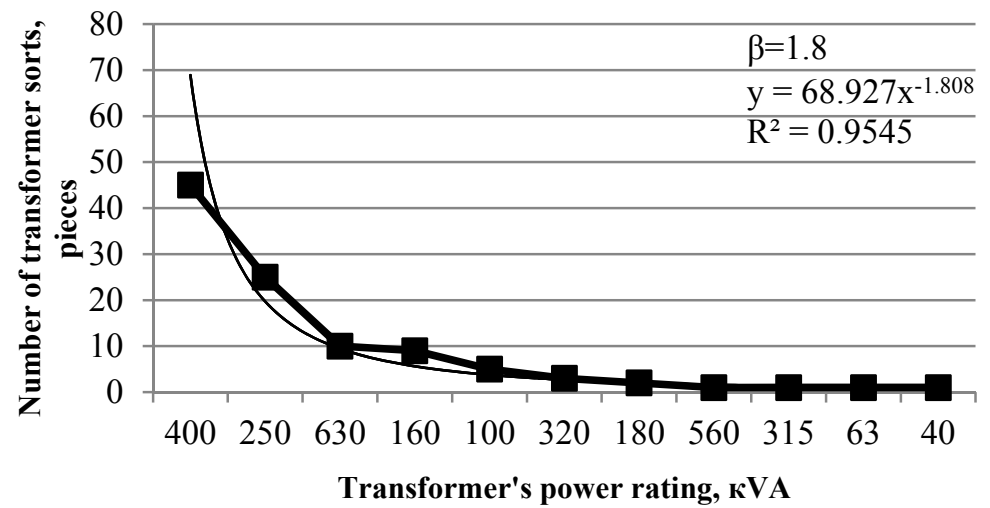

Figure 2: Transformer's rank allocation. 


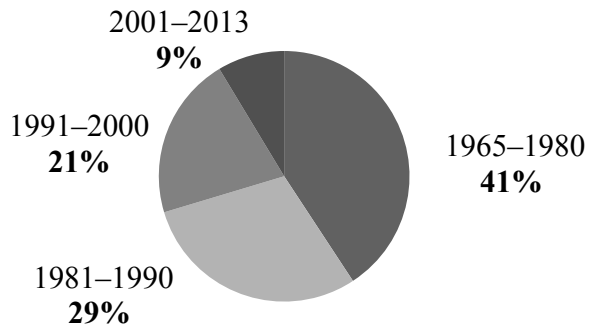

Figure 3: Age-specific structure of transformers installed at mono-town's substations.

Carrying out analysis of the aspectual and parametric technocoenosis structure (mono-town), we can organize an evolutional state estimation, using characteristic factors' values and its dynamics; we can develop recommendations for power equipment nomenclature and parametric structure corrections.

\section{Development of forecasting algorithm of mono-town power consumption}

It is important to have accurate forecasts of power consumption for a period of 1-5 years, in order to save energy resources and for the effective management of mono-town power consumption $[16,17]$. According to Russian experts, errors in the annual power consumption forecasts of $1 \%$ amount to $\$ 4$ billion in additional investments for construction generating facilities [18].

Increasing the requirements for the accuracy of power consumption forecasts of areas deals with the increasing restriction on allocated energy resources every year. The analysis of scientific papers, devoted to the methods of energy consumption forecasting, shows that the highest accuracy is reached by the use of a neural network of artificial intelligence [19].

However, in recent years a forecasting method has been developed on the basis of regression models as an alternative to the intellectual approach based on the method of support vectors $[18,20]$.

For the method of support vectors it is typical that the regression model parameters are determined by solving the quadratic programming problem, which has a unique solution $[11,21,22]$.

Forecasting accuracy is reached by the normalization and standardization of input data procedures for separate consumers groups (group I - enterprises, organizations, institutions, group II - population) and by using the cross-validation procedure. Approximation is made by means of the Gaussian kernel for the time series of group I, and for the group II - by means of a linear kernel [22, 23].

As a result, two forecasting models have been made for group I (error of $3 \%$ ) and for group II (error of $3.74 \%$ ), taking into account climatic factors without the characteristic exponent $\beta$ (fig. 4). 


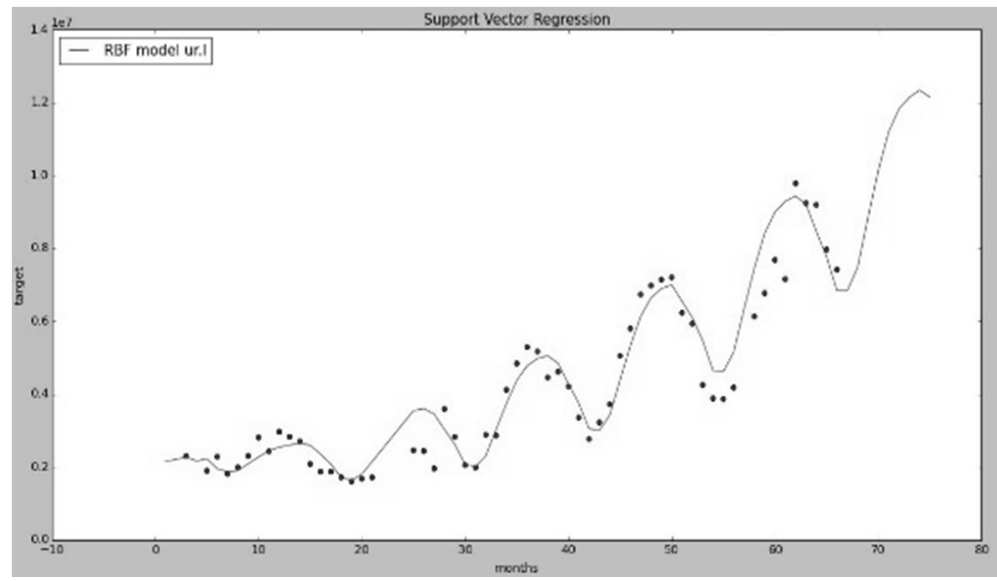

Group I

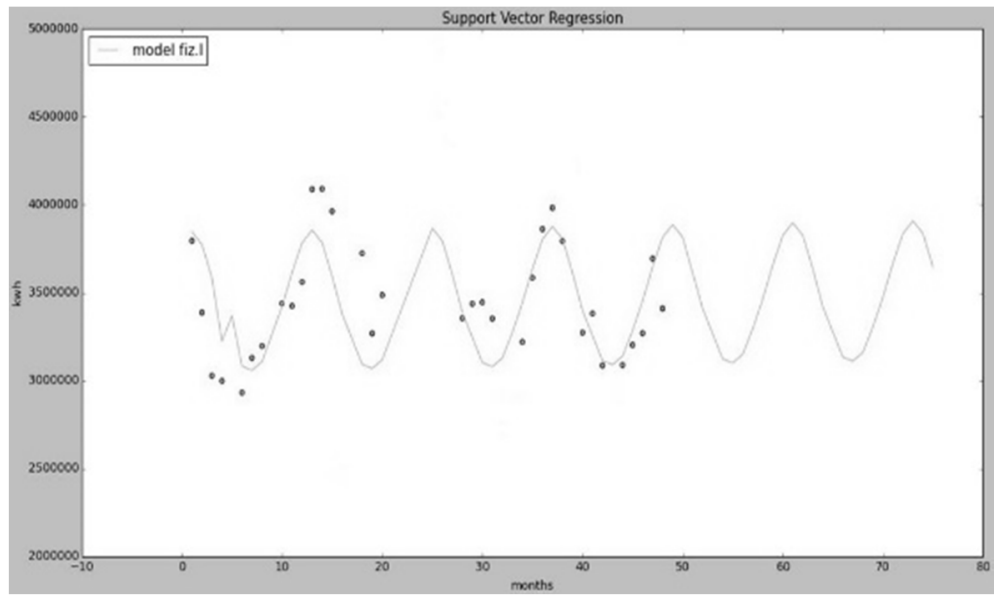

Group II

Figure 4: Energy consumption forecasting for groups I and II related to mono-towns.

A final energy consumption forecast can be obtained by means of combining single forecasts, that is, group I forecasting and group II forecasting.

The sustainable development model of a mono-town is to be based on target forecasting. The authors developed the algorithm for target forecasting performance by force of the correction of an energy consumption structure in a mono-town using rank and structural analysis (fig. 5).

The obtained model was tested using the example of a mono-town situated in the Sverdlovsk region.

The actual energy consumption data over a period of 2009-2013 years was used. 


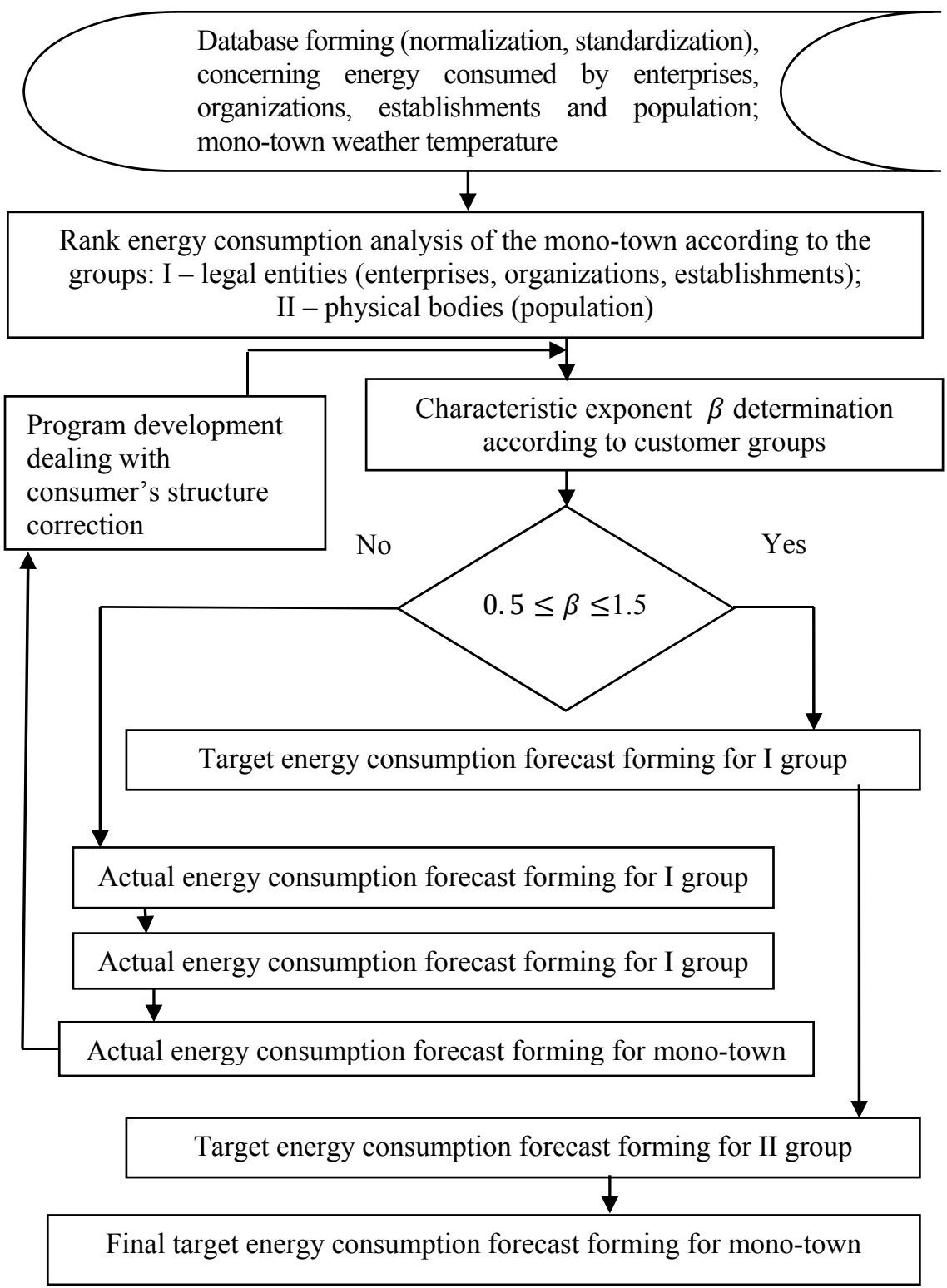

Figure 5: Target forecasting algorithm of mono-town's energy consumption. 


\section{Conclusion}

The rank and structural analysis of mono-towns' energy consumers forms an evolutional territory image. The technocoenosis approach is used as a basis for the analyzing process.

Using the characteristic exponent $\beta$ as an additional parameter during energy consumption forecasting we can take into account the structural features of technocoenosis. In addition, we have the ability to obtain both structural and actual forecasts owing to territorial correction program development and realization.

The implementation of target strategic forecasting (1-5 years) for mono-towns is an important instrument for efficient energy consumption and power supply system's monitoring (enterprises, organizations, establishments and population of a mono-town), leading to technocoenosis sustainability (viability).

\section{References}

[1] Uskova, T.V., Iogman, L.G., Tkachuk, S.N., Nesterov, A.N., Litvinova, N.Y. Mono-towns: Management of development [Text]. ISEDT, Academy of Sciences: Vologda, pp. 3-8, 2012.

[2] Hajrullov, D.S. Sustainable region development as a basis of national economy safety. Proc. of the $4^{\text {th }}$ All-Russia scientific conf. Information technologies in system of social and economic safety of Russia and its regions. Kazan, KFU, pp. 41-49, on April, 23-26rd 2012.

[3] Bobylev, S.N., Zubarevich, N.V., Soloveva, S.V., Vlasov, Ju.S. Sustainable development: methodology and measurement techniques. Moscow: Economy, 2011.

[4] 17 Indicators of sustainable development: framework and methodologies, UN commission on Sustainable Development, New York, 2001.

[5] Fedorova, S.V. Evolutionary approach to the management of municipalities' energy efficiency. Innovations and investments, pp. 207211, № 32014.

[6] Kuvshinov, M.A. Management concept of a sustainable development of municipalities' social and economic system. The young scientist, pp. 86-91, № 7 T.1 2011.

[7] Ikonnikova, O.V. Fundamental approaches to concept definition "the Sustainable development of rural territories". Agriculture Economy, pp. 349-352, № 1 (41) 2012.

[8] Gnatyuk, V.I. The law of technical coenosises optimum construction: the computer monograph. Publishing house TGU - the Center of system researches, 2005, 2011, http://gnatukvi.ru/ind.html.

[9] Fufaev, V.V. Cenological definition of parameters of electric energy consumption, reliability, installation and repair of region enterprises electric equipment. Moscow: the Center of system researches, 2000.

[10] Kudrin, B.I. Coenology. Technetics. Electrical/Electronic resource: http://www.kudrinbi.ru. 
[11] Support Vector Machines. Machine learning, http://www.statsoft.ru/home/textbook/modules/stmachlearn.html.

[12] Martynov, A.S., Artukhov, V.V., Vinogradov, V.G. Russia as a system: Integrated Analytical Web-Atlas, 1977. Access:

http://www.amr.vladimir.ru/text/kret3.htm.

[13] Armless, M.M., Farber, D.A. Psychophysiology. Dictionary. Moscow: PERSE (Psychological lexicon. Collegiate Dictionary in six volumes. Eds. Karpenko, L.A., Petrovsky, A.V.), 2006.

[14] Il'ichev, L.F., Fedoseyev, P.N., Kovalev, S.M., Panov, V.G. Dictionary of Philosophy. Main Edition: Moscow, Sov. Encyclopedia, 1983.

[15] Fedorova, S.V., Khudyakov, P. Yu. Structural and topological analysis of electric energy consumption of municipalities as the basis for the development of the regional energy efficiency strategy. Proc. of the $1^{\text {th }}$ Int. scientific conf. Energy Production and Management in the $21^{\text {st }}$ Century: Yekaterinburg, Russia, Vol. 1, WIT Transactions on Ecology and The Environment, Vol. 190, Energy Quest. pp. 407-417, on April, 23-25th 2014.

[16] Eroshenko S.A., Vinokurov K.V., Smolina A.Y. Electrical load forecasting. Proc. of the 1th Int. scientific conf. Energy Production and Management in the 21st Century: Yekaterinburg, Russia, Vol. 1 WIT Transactions on Ecology and The Environment, Vol. 190, Energy Quest, pp. 299-305, on April, 23-25th 2014.

[17] Kokin S.E., Pazderin A.V., Adarichev E.N., Ways of decreasing maximum and equalizing curve of big cities' power demand, Proc. of the $1^{\text {st }}$ Int. Conf. of Sustainable Power Generation and Supply, SUPERGEN '09: Nanjing, China, Article number 5347879, 6-7 April, 2009.

[18] Van Efen. Methodology of short-term improvement forecasting of electric energy consumption. Diss. auto abstract of the Cand. Tech. Sci: SaintPetersburg, 2013.

[19] Haykin, S. Neural networks: a complete course. Moscow: OOO "I.D. Williams", 2006.

[20] Polyakhov, N.D, Prikhodko, I.A., Van Efen. Forecasting of electric energy consumption for based on SVM method using evolutionary algorithms to optimize. Modern problems of science and education. №2 2013, http://www.science-education.ru/108-8962.

[21] SVM regression. MachineLearning.ru, http://www.machinelearning.ru/wiki/index.php.

[22] Cross-validation. MachineLearning.ru, http://www.machinelearning.ru/wiki/index.php.

[23] Scikit-learn. Machine Learning in Python, http://scikit-learn.org/stable.

[24] Nelson, R.R., Winter, S.J. An Evolutionary Theory of Economic Change. Moscow: Publishing House Case, 2002.

[25] Veblen T. Why economics is not an evolutionary discipline (1898). V: Origins. Studying of the economics as structure and process. Moscow: Ed. House HSE, 2006. 
[26] Popkov, V.V., Berg, D.B., Kuznetsov, P.O., authors foreword of Mayewskiy, V.I. Evolutionary dimension of strategic banking management: scientific ed. Ekaterinburg: Ural worker, 2002.

[27] Mayewskiy, V.I. Evolutionary theory and technological progress. Economic Issues. № 112001.

[28] Chaikovskiy, Y.V. Science of the life development. Experience of evolutionary theory. Moscow: KMK Scientific Press Ltd., 2006.

[29] Meyen, S.V. Problem of evolution directivity. Results of science and technology. Vertebrate Zoology. T.7 Problems of the theory of evolution. Moscow: Ed. VINITI, 1975.

[30] Chaitun, S.D. Development of scientific view of the relationship between the law of entropy increase and evolution. Concept of self-organization in a historical retrospective. Moscow: Ed. Science, 1994. 